\title{
Predicting the Effects of Discomfort Glare on Visual Performance of Pilots during Nighttime Flying
}

\author{
Yandan Lin*, Mengdi Dong, Yihong Liu, \\ Yaojie Sun \\ Institute for Electric Light Sources; Engineering \\ Research Center of Advanced Lighting Technology \\ Ministry of Education \\ Fudan University \\ Shanghai, China \\ *Corresponding Author: ydlin@ fudan.edu.cn
}

\author{
Weiqiao Yang, Feng Xiong \\ Semiconductor Lighting Technology Research Center \\ Shanghai, China
}

\begin{abstract}
Glare is a threat to aviation safety, as it can changed the adaptation status and visual function of pilots during nighttime flying. The evaluation of discomfort glare has been hotly debated in the past decades. This article presents two experiments, with three visual task involved, i.e. reaction time, detection threshold and discrimination threshold, to inspect Lin-Liu model's predicting effect of discomfort glare pilots encountered during nighttime flying, and also to explore how the discomfort glare affect the visual performance of the pilots. The results were analysed using repeated-measures ANOVA. It was confirmed that glare has a significant influence on the pilots' visual performance: under harsh luminous condition, visual performance got worse significantly. The evaluation result using Lin-Liu model, which expresses the perceived discomfort glare by means of deBoer rating $\left(\mathbf{R}_{\text {deBoer }}\right)$, showed a regressive relationship between deBoer rating and visual performance, which indicated a good prediction capability of Lin-Liu model in the influence of discomfort glare.
\end{abstract}

Keywords-discomfort glare; nighttime flying; visual performance; reaction time; discrimination threshold size;

\section{INTRODUCTION}

During nighttime flying, the cockpit of an aircraft is typically dim and pilots are adapted to the dim ambient environment, therefore pilot's vision is easily affected by external variations. Intense glare is a common problem. When pilot was exposed at strong lights suddenly, the adaptation status and the visual function would be changed, which would limit a pilot's response time and greatly increasing the risk of accidents [1].

Glare can be classified as disability glare or discomfort glare. For disability glare, it has been fairly well defined based on the physiology of the human eye and the behavior of light as it enters the ocular media $[2,3]$. On the other hand, discomfort glare is more about a psychological response, and it's more difficult to solve than issues with disability glare. The evaluation of discomfort glare was hotly debated in the past decades [4].
The deBoer rating scale is the most widely adopted scale used to evaluate discomfort glare. It's a nine-point scale with adjectives near the odd numbers. A larger value on the deBoer rating scale represents less perceived discomfort glare [5]. Based on the deBoer rating scale, many models for discomfort glare have been developed [58]. Recently, a new model, Lin-Liu model, predicting discomfort glare caused by LED light source has been published [9], as shown in Eq. (1), which was also based on the deBoer rating scale.

$$
R_{\text {deBoer }}=3.45-\log _{10}\left(\frac{\left(L_{g} \times \omega\right)^{2.21}}{L_{b}^{1.02} \times \theta^{1.62}}\right)
$$

According to the Lin-Liu model, perceived discomfort glare expressed by means of a deBoer rating $\left(\mathrm{R}_{\text {deBoer }}\right)$ is related to the average glare source luminance $\left(\mathrm{L}_{\mathrm{g}}\right)$, the background luminance $\left(\mathrm{L}_{\mathrm{b}}\right)$, the solid angle of the glare source from the perspective of the viewer $(\omega)$ and the angle between the glare source and the line of sight $(\theta)$.

The aim of this article is to inspect Lin-Liu model's predicting effect of discomfort glare pilots encountered during nighttime flying, and also to explore how the discomfort glare affect the visual performance of the pilots. Pilots' visual performance was characterized by three visual tasks, which were reaction time of detecting the target outside the cockpit, the detection and discrimination threshold size of the Landolt ring on the display screen. Discomfort glare varied glare source luminance, background luminance or solid angle of the glare source from the perspective of the viewer.

\section{EXPERIMENT 1: REACTION TIME}

\section{A. Method}

An experiment was carried out to investigate the influence of discomfort glare on the reaction time of detecting the target outside the cockpit. The apparatus, shown in Fig .1, consisted of a cockpit model, an outside background projection screen and glare source. The apparatus was setup to simulate the scene that the pilot 
encountering the discomfort glare from the lighting installation at the airport when landing, and the whole apparatus was put in a dark room which ca avoid interference of other light outside.

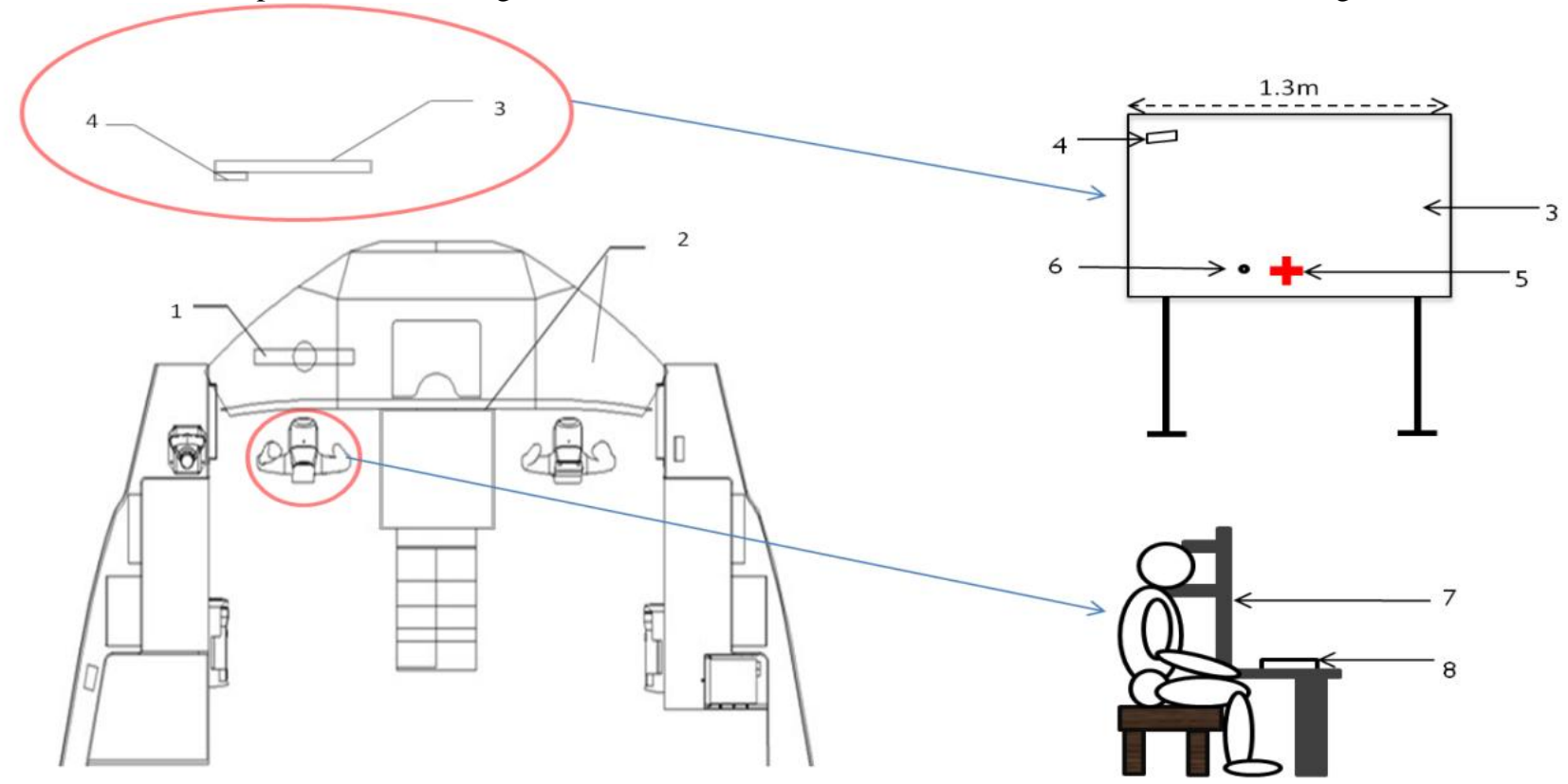

Figure 1. The apparatus used to measuring the reaction time (1. display screen, 2.operation panel, 3. projection screen, 4. glare source, 5. sight fixing point, 6.task target, 7. chin rest, 8. keyboard.)

The cockpit model was made according to the real condition with the proportion of 1:1, in which participants sat and reacted. In front of the cockpit model, a big projection screen was set to mimic the background environment outside of the cockpit, with a distance of $4 \mathrm{~m}$ to the participants. At the middle bottom of the screen, there was a red cross mark, used for fixing participants' sight to avoid them looking straight at the glare source, which might result in directly influence on visual function. The glare source used in the experiment was a LED module with the nominal power of $23 \mathrm{~W}$. It was hung in the upper left corner of the projection screen and the observing angle was $10^{\circ}$ which was in accordance with the real situation.

A computer software was independently programmed to estimate and record the reaction time. The observing target was generated by the computer software, and it was a black dot with a size of 12.9 minute of arc and eccentric angle of $2^{\circ}$. The target was projected on the projection screen, mimicking the obstacle outside the cockpit. During the experiment, participants were asked to press the button on the wireless keyboard after he/she perceiving the target appears as soon as possible. The reaction time of each hit was recorded. Before the target appearing, there is a sound signal to draw the observers' attention to look at the sight fixation.

The parameter setup in detail is shown in Table I. Three parameters related to the glare rating were selected as variables. With different combinations of the three variables, there were 12 lighting conditions for test in total.

TABLE I. PARAMETERS SETUP IN THE RESEARCH ON THE EFFECT OF GLARE ON REACTION TIME

\begin{tabular}{|l|c|c|}
\hline \multicolumn{2}{|l|}{ Parameters } & Values \\
\hline Constants & Adaptation time(min) & 20 \\
\hline
\end{tabular}

\begin{tabular}{|c|c|c|}
\hline \multicolumn{2}{|c|}{ Parameters } & Values \\
\hline & CCT of glare source $(\mathrm{K})$ & 6500 \\
\hline & Glare source angle $\left(\theta:^{\circ}\right)$ & 10 \\
\hline & Observing distance $(\mathrm{m})$ & 4 \\
\hline & Observing way & Binocular \\
\hline & Eccentric angle $\left(^{\circ}\right)$ & 2 \\
\hline & Size of the target(min of arc) & 12.9 \\
\hline \multirow{3}{*}{ Variables } & $\begin{array}{l}\text { Glare source luminance }\left(\mathrm{L}_{\mathrm{g}} \text { : }\right. \\
\left.\mathrm{cd} / \mathrm{m}^{2}\right)\end{array}$ & $\begin{array}{c}800 \\
/ 8000 / 80000\end{array}$ \\
\hline & $\begin{array}{l}\text { Background luminance }\left(\mathrm{L}_{\mathrm{b}} \text { : }\right. \\
\left.\mathrm{cd} / \mathrm{m}^{2}\right)\end{array}$ & $1 / 8$ \\
\hline & $\begin{array}{l}\text { The solid angle of the glare } \\
\text { source }(\omega: \text { sr })\end{array}$ & $\begin{array}{l}0.8 \times 10^{-5} / \\
3.1 \times 10^{-5}\end{array}$ \\
\hline
\end{tabular}

10 test participants were recruited from staff and students of Fudan University: 4 were female and 6 were male, and of approximate mean age 22 years. All test participants had normal (or corrected to normal) visual acuity as tested using a Snellen E chart. Normal colour vision was confirmed using the Ishihara Pseudoisochromatic Plates.

At the start of each test session, 20 minutes was allowed for adaptation to the low light level. Each observer was informed the whole procedure of the experiment and asked to complete practices with the testing software before the formal tests to avoid the practice effect. Then, a lighting condition was setup, following which the participants complete one trial and the testing software recorded the reaction times. Prior to next lighting condition, there was a period of resting time to avoid visual fatigue. The presentation order of the lighting conditions was randomized. Every participant carried out 12 trials. The whole experiment was made up of adaptation stage, 
measurement stage and resting, taking about 60 minutes in total.

\section{B. Results}

Fig .2 shows the mean reaction time of the 10 participants, for 12 lighting conditions. In Fig .2, horizontal coordinate is the glare source luminance and vertical coordinate is the mean reaction time of the 10 participants.
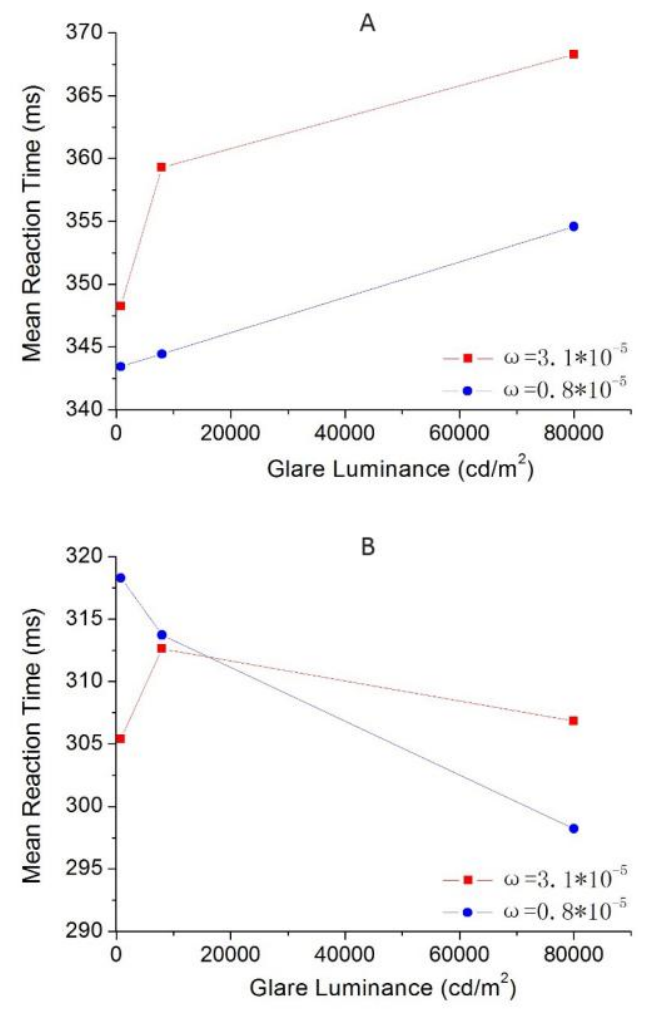

Figure 2. Results of experiment 1: Reaction time. Mean reaction time plotted against the glare luminance for two background luminance and two solid angle of the glare source. (A: background luminance $1 \mathrm{~cd} / \mathrm{m}^{2} ; \mathrm{B}$ : background luminance $8 \mathrm{~cd} / \mathrm{m}^{2}$ )

For the low background luminance $\left(1 \mathrm{~cd} / \mathrm{m}^{2}\right)$, the data exhibits a similar trends: mean reaction time increases at higher glare luminance, and mean reaction time for the bigger solid angle is longer than that for the smaller solid angle. While, for the higher background luminance $(8$ $\mathrm{cd} / \mathrm{m}^{2}$ ), there's no obvious trend between mean reaction time and glare luminance for both solid angle.

Statistical analyses of these results were carried out with the reaction time of the 10 participants using repeated-measures ANOVA. The analysis result demonstrated that, for the $1 \mathrm{~cd} / \mathrm{m} 2$ background luminance, the effects of glare source luminance $(\mathrm{F}=3.403$, $\mathrm{P}=0.034<0.05)$ and solid angle $(\mathrm{F}=5.161, \mathrm{P}=0.023<0.05)$ were significant, while, for the $8 \mathrm{~cd} / \mathrm{m} 2$ background luminance, the effects of glare source luminance and solid angle were not suggested to be significant. As to the effect of background luminance, the repeated-measures ANOVA suggests this is significant $(\mathrm{F}=192.739, \mathrm{P}<0.05)$, for both two solid angle, with higher background luminance leading to a shorter mean reaction time.

\section{EXPERIMENT 2: DETECTION AND DISCRIMINATION}

\section{A. Method}

Experiment 1 used reaction time of detecting the target outside the cockpit to characterize the visual performance. In experiment 2 , the detection and discrimination threshold size of the Landolt $\mathrm{C}$ on the display screen was involved.

The apparatus in experiment 2 was the same with that in experiment 1 . The glare source was still the LED module with an observing angle of $10^{\circ}$. In experiment 2 , the solid angle of glare source and background luminance were set as constant, different with that in experiment 1 .

The Landolt $\mathrm{C}$ was used as target for the detection and discrimination task, and it was displays on a LCD display screen (marked with No. 1 in Fig .1), with a distance of $0.7 \mathrm{~m}$ to participants' eyes. The size of Landolt $\mathrm{C}$ could increase or decrease gradually, and participants were asked to detect the Landolt $\mathrm{C}$ and the discriminate the direction of the Landolt $\mathrm{C}$. The detection threshold size and discrimination threshold size were recorded.

The parameter setup in detail is shown in Table II. Glare source luminance and target (Landolt C) luminance were selected as variables. With different combinations of the three variables, there were 25 lighting conditions for test in total.

TABLE II. PARAMETERS SETUP IN THE RESEARCH ON THE EFFECT OF GLARE ON DETECTION AND DISCRIMINATION THRESHOLD SIZE.

\begin{tabular}{|c|c|c|}
\hline Parameters & Values \\
\hline \multirow{5}{*}{ Constants } & Adaptation time $(\mathrm{min})$ & 20 \\
\cline { 2 - 3 } & CCT of glare source $(\mathrm{K})$ & 6500 \\
\cline { 2 - 3 } & Glare source angle $\left(\theta:^{\circ}\right)$ & 10 \\
\cline { 2 - 3 } & $\begin{array}{c}\text { The solid angle of the } \\
\text { glare source }(\omega: \mathrm{sr})\end{array}$ & $3.1 \times 10^{-5}$ \\
\cline { 2 - 3 } & $\begin{array}{c}\text { Observing way } \\
\text { Variables }\end{array}$ & $\begin{array}{c}\text { Background } \\
\text { luminance }\left(\mathrm{L}_{\mathrm{b}}: \mathrm{cd} / \mathrm{m}^{2}\right)\end{array}$ \\
\cline { 2 - 3 } & $\begin{array}{c}\text { Glare source } \\
\text { luminance }\left(\mathrm{L}_{\mathrm{g}}: \mathrm{cd} / \mathrm{m}^{2}\right)\end{array}$ & $1000 / 5000 / 10000$ \\
& $\begin{array}{c}\text { Target luminance }\left(\mathrm{L}_{\mathrm{t}}:\right. \\
\left.\mathrm{cd} / \mathrm{m}^{2}\right)\end{array}$ & $1 / 30000 / 80000$ \\
\hline
\end{tabular}

10 test participants were recruited from staff and students of Fudan University: 4 were female and 6 were male, and of approximate mean age 22 years. These participants also took part in the Experiment 1.

At the start of each test session, 20 minutes was allowed for adaptation to the low light level. Each observer was informed the whole procedure of the experiment and asked to complete practices with the testing software before the formal tests to avoid the practice effect. Then, a lighting condition was setup, following which the participants were asked to press a button on the keyboard, until he/she could or could not detect the Landolt $\mathrm{C} /$ discriminate the direction of Landolt $\mathrm{C}$. The presentation order of the lighting conditions was randomized. Every participant carried out 25 trials. 


\section{B. Results}

Fig .3 shows the mean detection threshold size and mean discrimination threshold size of the 10 participants, for 25 lighting conditions.

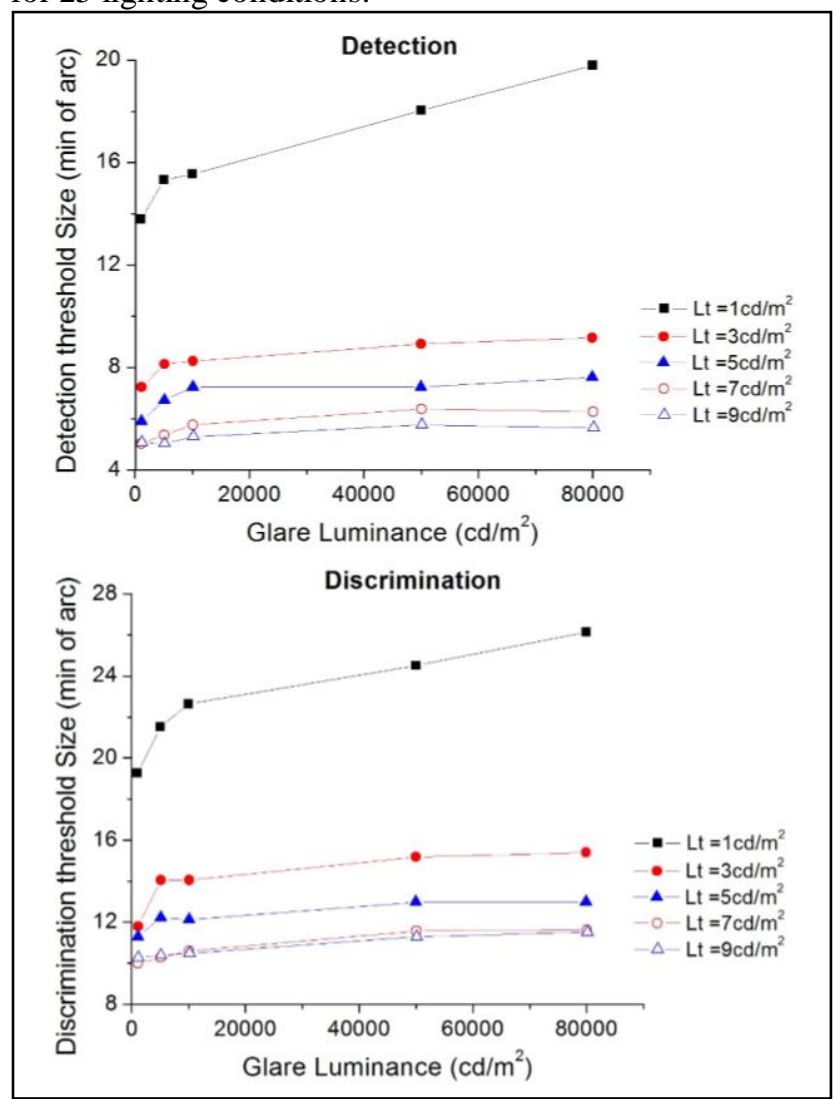

Figure 3. Results of experiment 2: Detection/Discrimination threshold size. Mean threshold size plotted against the glare luminance for five target luminance.

The trends exhibited by detection threshold size is similar to those by discrimination threshold size: higher target luminance lead to lower threshold size. The threshold size of discrimination task is much larger than that of detection task. It's expectable as the difficulty of discrimination is much harder than detection.

For both detection and discrimination, the threshold size of $1 \mathrm{~cd} / \mathrm{m}^{2}$ target luminance is clearly larger than that of other four target luminance, and the difference decreases as the target luminance increase. As to the influence of glare luminance, the detection and discrimination threshold size increase with glare luminance, and there is a plateau region where the glare luminance is larger than $50000 \mathrm{~cd} / \mathrm{m}^{2}$.

As with experiment 1 , statistical analyses were carried out for the threshold size of the 10 participants using the repeated-measures ANOVA. This suggests that effects of glare luminance are significant for detection $(\mathrm{F}=5.069$, $\mathrm{p}<0.001)$ and discrimination $(\mathrm{F}=4.489, \mathrm{p}=0.001)$ task. The effects of target luminance are also significant for detection $(\mathrm{F}=96.090, \quad \mathrm{p}<0.001)$ and discrimination $(\mathrm{F}=67.496, \mathrm{p}<0.001)$ task.

\section{DISCUSSION}

The results (Fig .2 and Fig .3) indicate that glare affects the visual performance, both for the reaction time of detecting the target outside the cockpit and the detection and discrimination threshold size of the Landolt ring on the display screen. With higher glare luminance, lower background luminance or larger solid angle of the glare source, visual performance got worse significantly. In order to predict the effect of discomfort glare, we use LinLiu model to evaluate the discomfort glare in these two experiments.

\section{A. Experiment 1: Reaction time}

The results of experiment 1 show that the mean reaction time for the low background luminance $\left(1 \mathrm{~cd} / \mathrm{m}^{2}\right)$ increases at harsh luminous conditions, while for the higher background luminance $\left(8 \mathrm{~cd} / \mathrm{m}^{2}\right)$, this trend is not so obvious. The data for the lower background luminance $\left(1 \mathrm{~cd} / \mathrm{m}^{2}\right)$ is evaluated by Lin-Liu model, expressed by means of deBoer rating (Fig .4).

According to the definition of deBoer rating, it's a nine point scale where 1 represents "Unbearable" glare, 3 is "Disturbing", 5 is "Just Admissible", 7 is "Satisfactory" and 9 is "Unnoticeable". Calculation with Lin-Liu model shows that mean reaction time increases with deBoer rating decreases, and this is predictable, as the higher deBoer rating means a better lighting condition, resulting in faster reaction and shorter reaction time.

The relationship between deBoer rating and reaction time is fitted by linear function. The result of Linear Fitting suggests a regressive relationship between reaction time and deBoer rating. The result indicates a good prediction of Lin-Liu model on the influence of glare on reaction time.

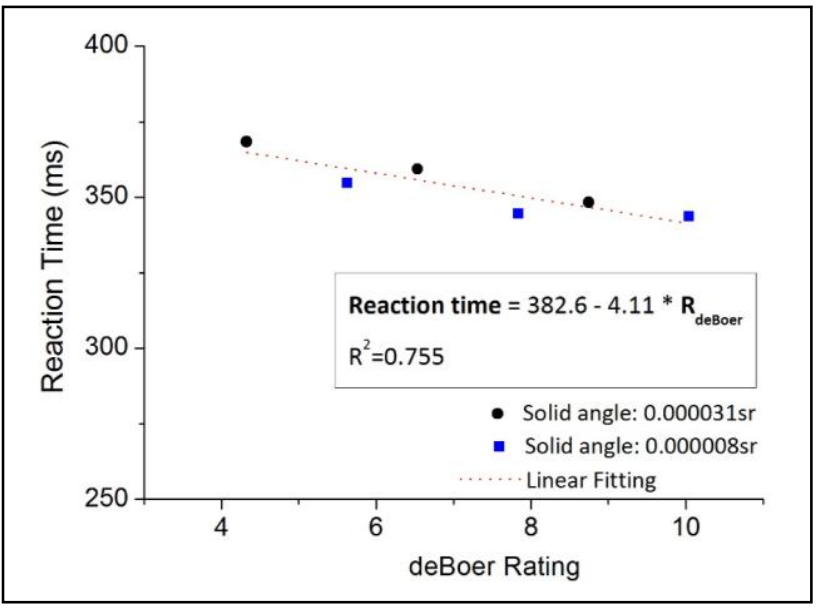

Figure 4. Relationship between reaction time and deBoer rating evaluated by Lin-Liu model (background luminance $1 \mathrm{~cd} / \mathrm{m}^{2}$ ).

\section{B. Experiment 2: Detection and Discrimination}

In experiment 2, glare source luminance is the only variable of glare condition, and the results suggest that higher target luminance lead to larger threshold size, both for the detection and discrimination. Same as experiment 1 , the data of experiment is evaluated by Lin-Liu model (Fig .5).

The evaluation result using Lin-Liu model also shows a linear regressive relationship between threshold size and deBoer rating, both for detection and discrimination, which exhibits a similar trend as that in Fig .5. According to the results, $\mathrm{R}^{2}$ of Linear Fitting is larger than 0.9 for most trial, 
and Linear Fitting suggests a good prediction ability of Lin-Liu model on the experiment result.

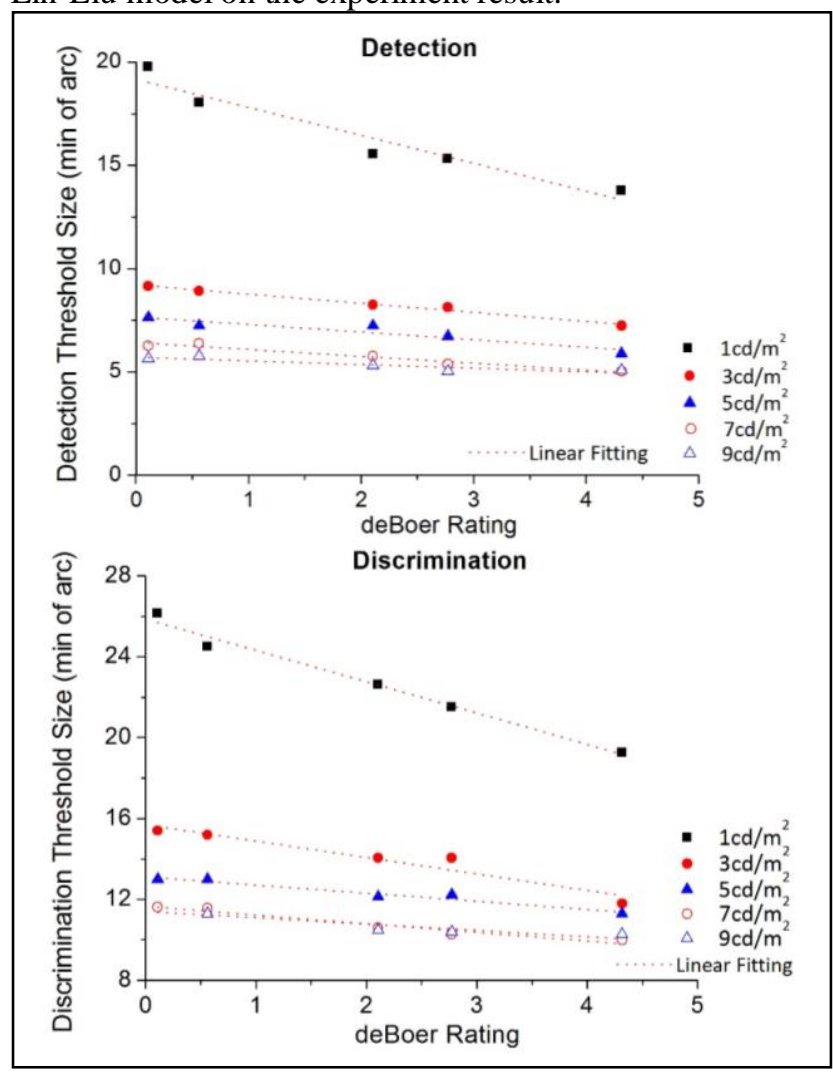

Figure 5. Relationship between threshold size (for different target luminance) and deBoer rating evaluated by Lin-Liu model

\section{CONCLUSION}

This article reports investigation of influence of glare on pilots' visual performance during nighttime flying, using three visual tasks: reaction time detecting the target outside the cockpit, the detection and discrimination threshold size of the Landolt ring on the display screen. The results indicate the significant influence of glare on the pilots' visual performance. With higher glare luminance, lower background luminance or larger solid angle of the glare source, visual performance got worse significantly. The influence of discomfort glare was evaluated using a Lin-Liu model, which expresses the perceived discomfort glare by means of deBoer rating $\left(\mathrm{R}_{\mathrm{deBoer}}\right)$. The analysis using data from reaction time task, detection and discrimination task indicates a good prediction capability of Lin-liu model in the influence of glare on visual performance: lower $\mathrm{R}_{\mathrm{deBoer}}$, corresponding a harsher luminous condition, resulting in worse visual performance.

\section{ACKNOWLEDGEMENT}

This work was carried out as part of the National Basic Research Program of China (973) (Project NO. 2010CB734102) and project funded by Ministry of Science and Technology of China (Project No. 2011BAE34B01)

\section{REFERENCES}

[1] B. Yang, Y. Lin, and Y. Sun, "Transient effects of harsh luminous conditions on the visual performance of aviators in a civil aircraft cockpit.” Applied Ergonomics, vol 44, 2013, pp185-191.

[2] W. S. Stiles, "Discussion on disability glare" presented at the CIE meeting, Scheveningen, The Hague, 1939.

[3] J. J. Vos, "On the cause of disability glare and its dependence on glare angle, age and ocular pigmentation," Clin. Exp. Optom, vol 86, 2003, pp363-370.

[4] D. Fisher, "Discomfort glare in interiors" presented at the First International Symposium on Glare, Orlando, Florida, New York, 24-25 Oct. 1991.

[5] J. B. deBoer, D. A. Schreuder, "Glare as a criterion for quality in street lighting," Trans.Illum.Eng. Soc, vol 32, 1967, pp117-135.

[6] H. J. Schmidt-Clausen, J. T. H. Bindels, "Assessment of discomfort glare in motor vehicle lighting," Lighting Res. Tech, vol 6, 1974, pp79-88.

[7] J. D. Bullough, J. A. Brons, R. Qi, and M. S. Rea, "Predicting discomfort glare from outdoor lighting installations," Lighting Res. Tech, vol 40, 2008, pp225-242.

[8] J. D. Bullough, K. Sweater-Hickcox, and N. Narendran, "A method for estimating discomfort glare from exterior lighting system," Alliance for Solid-State Illumination Systems and Technologies 9, 2011, pp1-7.

[9] Y. Lin, Y. Liu, and Y. Sun, "Model predicting discomfort glare caused by LED road lights," Optics express, vol 22, DOI: 10.1364/OE.22.018056.

[10] Y. Liu, "Investigation of the effects of discomfort glare on visual performance of pilots during nighttime flying," Fudan University, 2007. 